Quelles sont les exigences de mes patients vis-à-vis de moi, médecin de famille, et quels souhaits cachent-elles?

Dengler-Voss, Carolin ; Voss, Rödiger

Posted at the Zurich Open Repository and Archive, University of Zurich ZORA URL: https://doi.org/10.5167/uzh-102538

Journal Article

Published Version

Originally published at:

Dengler-Voss, Carolin; Voss, Rödiger (2014). Quelles sont les exigences de mes patients vis-à-vis de moi, médecin de famille, et quels souhaits cachent-elles? PrimaryCare : Journal Suisse de Médicine de Premier Recours, 14(18):289-291. 


\section{Quelles sont les exigences de mes patients vis-à-vis de moi, médecin de famille, et quels souhaits cachent-elles?}

\begin{abstract}
Introduction
Les médecins de famille sont confrontés à de nombreux patients au quotidien. II s'agit d'individus et de fait, ils n'ont pas le même niveau d'information et ont des attentes et des exigences différentes vis-à-vis de leur médecin. En raison de sa complexité et de sa durabilité, la relation entre le médecin de famille et le patient dépasse largement un simple modèle de service [1]. Les connaissances apportées par cette étude doivent permettre de mieux cerner les motivations des patients et d'optimiser la relation entre le médecin de famille et le patient. II s'agit de la première utilisation de la technique du laddering pour déduire les exigences des patients vis-à-vis de leur médecin de famille en Suisse.
\end{abstract}

\section{Méthodologie}

La technique du laddering, issue de la recherche en marketing américaine, est utilisée dans cette étude afin de mettre en évidence les relations entre les exigences de performance des patients vis-à-vis des médecins de famille et les valeurs personnelles qui y sont associées. Le laddering consiste à créer une chaîne, avec comme maillon initial une compétence ou qualification des médecins, comme maillon intermédiaire les conséquences (bénéfices) associées à la compétence et comme maillon final la valeur qui se cache derrière. Dans ce contexte, il a été demandé aux patients quelles étaient les compétences et qualifications dont devaient faire preuve les médecins de famille lors d'une consultation. Le bénéfice des attributs cités («Pourquoi la propriété/aptitude citée est-elle essentielle?») et les valeurs qui se cachent derrière ont ensuite été déterminés. Voici un exemple pour illustrer une conversation entre l'interviewer (I) et le patient (P) (voir tableau 1):

Tableau 1

Exemple d'interview de laddering.

I: Quelles sont les compétences et les qualifications que vous attendez d'un médecin de famille lors d'une consultation?

P: Des connaissances spécialisées, des aptitudes de communication (attributs).

I: Pourquoi jugez-vous essentiel qu'un médecin ait des connaissances spécialisées?

$P$ : Je veux être bien soigné. Le médecin doit faire le bon diagnostic (bénéfice).

I: Pourquoi voulez-vous être bien soigné?

$P$ : Je veux être rapidement rétabli ... (bénéfice).

I: Pourquoi voulez-vous être rapidement rétabli?

P: Après tout, chacun veut être en bonne santé, n'est-ce pas? ... (valeur)

A I'aide d'un logiciel, une «Hierarchical Value Map» (HVM) a été générée à partir des données dont le contenu a été analysé. II s'agit d'une représentation graphique des principales chaînes de laddering de tous les individus interrogés, permettant de comprendre et d'expliquer leur comportement et leurs souhaits.

\section{Résultats}

Au total, 35 personnes (19 femmes et 16 hommes) de Suisse alémanique ont été interrogées. Toutes les personnes interrogées avaient une activité professionnelle et étaient âgées de 40 à 65 ans. Dans le cadre de l'échantillonnage théorique, ce groupe d'âge a été choisi car les individus de cet âge, en raison de leur vécu, ont déjà accumulé suffisamment d'impressions et d'exigences lors du contact avec des médecins. Ont uniquement été intégrées dans l'échantillon les personnes ayant eu au minimum trois contacts avec leur médecin de famille au cours des 2 dernières années. L'âge moyen des participants était d'environ 52 ans. Près de $80 \%$ des personnes interrogées ont eu plus de trois consultations par an chez leur médecin de famille.

Précisions pour l'interprétation de la HVM: La taille des cercles correspond à la fréquence à laquelle les attributs, conséquences et valeurs ont été cités par les personnes interrogées. L'épaisseur des lignes de connexion entre les attributs, les conséquences et les valeurs représente la fréquence à laquelle les concepts en question ont été nommés ensemble. Sont représentées sur la HVM les connexions entre les attributs (cercles blancs), les conséquences (cercles gris) et les valeurs (cercles noirs) (voir fig. 1).

Sur la HVM, il apparaît clairement que les patients attendent des médecins de famille sept principales compétences et qualifications: connaissances spécialisées, compétence communicationnelle, amabilité, empathie, proactivité, expérience professionnelle et patience. Ces résultats relatifs aux exigences des patients n'ont rien de fondamentalement nouveau. Des études similaires réalisées en Suisse [2], en Angleterre [3] et en Allemagne [4] ont révélé des attributs semblables. Les connexions avec les bénéfices pour les patients et avec les valeurs sont particulièrement intéressantes et au même titre que les attributs, elles sont discutées dans les lignes qui suivent.

\section{Discussion}

Dans notre enquête, les connaissances spécialisées d'un médecin généraliste étaient l'attribut prédominant. Dans le cadre d'une enquête représentative réalisée en Suisse, Schläpfer [2] rapporte également l'importance particulière de cet attribut. Une solide formation pré-graduée, ainsi que la formation continue et la formation post-graduée constituent dès lors une composante essentielle de l'activité médicale. Pour les patients interrogés, être capable d'orienter le patient vers un spécialiste compétent fait également partie des connaissances spécialisées. Ainsi, les participants ne considèrent pas le médecin de famille comme un «je-saistout». Un participant a par exemple expliqué que les connaissances existantes se ressentaient lorsque le traitement se déroulait sans accroc. La perception de la compétence médicale a de vastes conséquences: lorsqu'un patient est convaincu de la compétence du médecin, il est prêt à accepter le manque de sympathie de l'assistante médicale, même si ce comportement le gêne beaucoup [6]. D'après nos participants, les médecins généralistes peuvent 


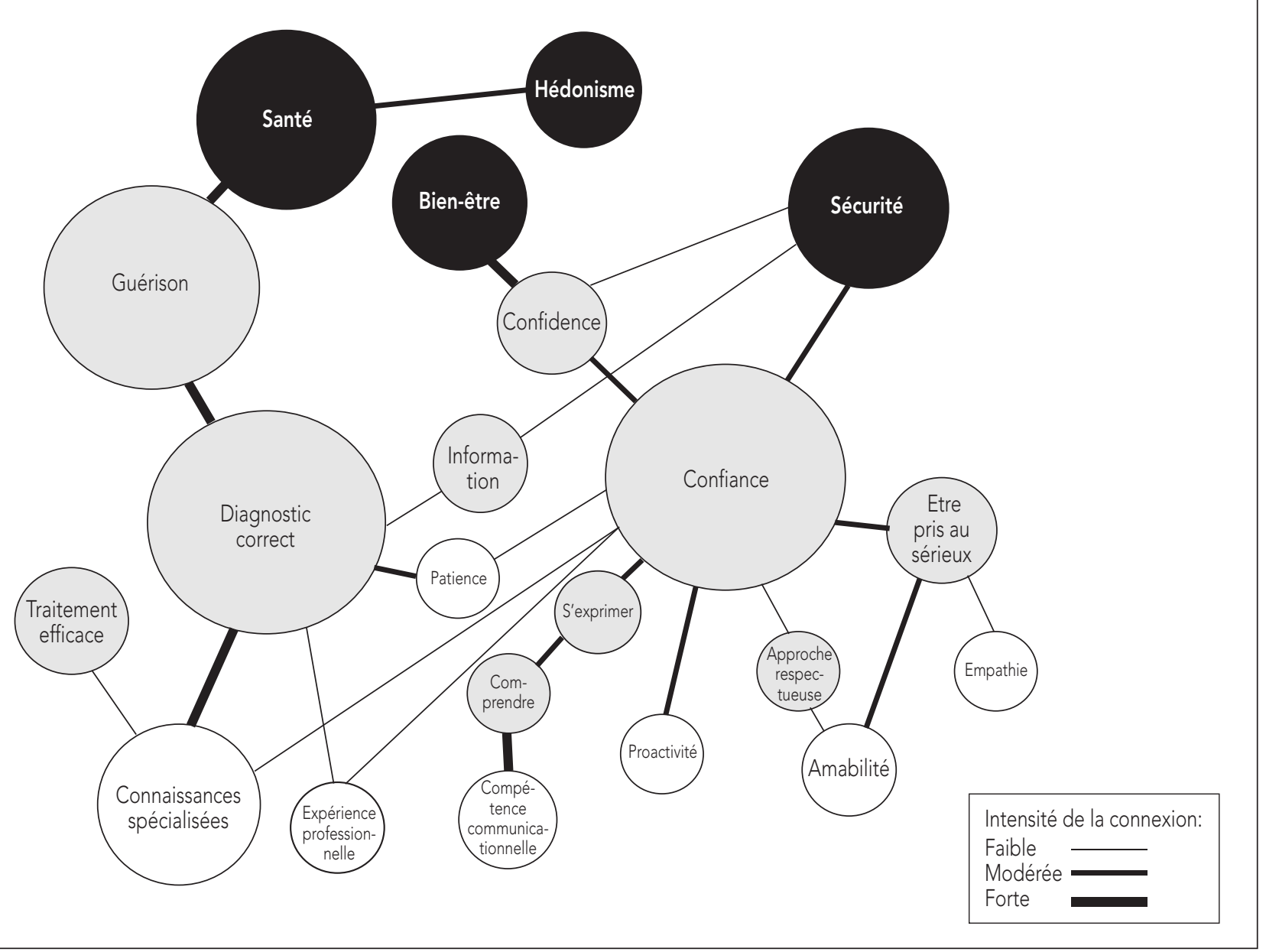

Figure 1

«Hierarchical Value Map» (HVM) des patients.

manifester leur compétence en se référant aux résultats actuels de la recherche. Les volontaires les plus jeunes de notre collectif attachaient également de l'importance à la présence sur internet de leur médecin, ainsi qu'à leur CV. II est dès lors utile pour le médecin généraliste de travailler sur ces points. En parallèle, accrocher dans le cabinet les certificats de formation post-graduée obtenus peut également permettre de faire état des connaissances spécialisées actuelles.

Les patients estiment que grâce à ses connaissances spécialisées, le médecin peut permettre un traitement efficace, ce qui revient avant tout à faire preuve d'efficience en termes de temps. Les patients n'aiment pas devoir patienter longtemps dans la salle d'attente lorsqu'ils ont rendez-vous. Le fait qu'un médecin de famille possède une longue expérience professionnelle produit le même effet que les connaissances spécialisées. Les résultats de l'étude de Neuenschwander/Riedel [1] ont montré qu'une longue expérience faisait partie des critères définissant un bon médecin de famille. D'après les participants, l'expérience professionnelle et les connaissances spécialisées aident à poser le bon diagnostic et donc à initier le bon traitement, ce qui conduit à la guérison, puis à la valeur «santé».

Lors de la consultation, une bonne compétence communicationnelle est attendue de la part du médecin généraliste. Les patients veulent comprendre les descriptions du médecin: d'après eux, I'utilisation du jargon médical devrait être évitée et les explications devraient être formulées de manière intelligible. Les termes médicaux latins sont contre-productifs car ils ne font pas partie du langage utilisé par le patient. Peters [6] souligne que des études linguistiques de discours ont montré qu'une bonne communication était à l'origine d'une meilleure observance. Les patients veulent non seulement comprendre les décisions médicales, mais ils veulent également en discuter avec le médecin généraliste. Ce dernier devrait dès lors pouvoir interpréter des informations et comprendre des opinions. Les médecins peuvent acquérir ou améliorer ces compétences par le biais de jeux de rôles et d'autres méthodes d'exercice avec des collègues expérimentés, de formateurs en communication ou d'offres de formation post-graduée spécifiques [7].

La patience est un autre attribut que devrait posséder le patient de famille, ce qui signifie qu'il devrait consacrer du temps aux patients et à leur traitement. De cette manière, il peut cerner le souhait de son patient et parvenir à poser le bon diagnostic. Par ailleurs, la patience favorise la mise en confiance du patient, comme en témoigne la déclaration d'une patiente: «Lorsqu'il prend du temps, cela signifie qu'il s'intéresse à moi et $\mathrm{m}$ 'amène à lui faire confiance.» A côté des connaissances spécialisées et de la compétence communicationnelle, l'amabilité est l'un des trois principaux attributs pour les patients et il s'agit donc d'un aspect central de tout traitement respectueux. Lorsqu'un médecin est aimable et fait en plus preuve d'empathie, les patients se sentent pris au sérieux. Les 
résultats de recherche de Neuenschwander et Riedel [1] montrent également que le médecin doit consacrer du temps aux patients et les prendre au sérieux. Ce faisant, les patients considèrent le médecin comme un partenaire au sein d'une relation d'égalité. En conséquence, les patients développent de la confiance à l'égard de leur médecin et peuvent s'ouvrir et se confier à lui, ce qui donne naissance à un sentiment de bien-être.

L'attribut «proactivité» est moins prononcé. Cet attribut englobe le fait que le médecin questionne activement le patient et qu'il fasse preuve d'un engagement affiché. Autrement dit, le médecin doit activement interroger le patient sur ses conceptions et ses attentes et il doit témoigner de l'intérêt pour une démarche commune. Donner-Banzhoff [8] résume ces critères sous l'expression «approche médicale centrée sur le patient».

\section{Message clé}

Par le biais des connaissances spécialisées, de la patience et de l'expérience de leur médecin de famille, les patients espèrent qu'un diagnostic le plus exact possible soit posé. L'objectif pour le patient est d'obtenir la guérison et au final, de recouvrer la santé. La santé est l'une des valeurs centrales des patients. Cela était à prévoir, étant donné que l'objectif primaire du traitement est I'amélioration de l'état de santé du patient. Après une maladie ou un accident, les individus veulent retrouver le plus rapidement possible une vie normale, pouvoir passer du temps avec leur famille et leur entourage social et en profiter. Lorsqu'ils sont en bonne santé, les individus peuvent aspirer à la joie, à la délectation et au plaisir (hédonisme). Par ailleurs, la pose d'un diagnostic correct offre la possibilité d'informer le patient sur son état, ce qui lui confère un sentiment de sécurité. Une fois le diagnostic établi, les patients attendent du médecin de famille qu'il leur fournisse des informations détaillées et suffisantes.

A côté du diagnostic adéquat, la confiance constitue le principal bénéfice pour les patients. Lorsque les patients développent de la confiance, ils peuvent s'ouvrir et communiquer librement, même $s^{\prime} i l$ est question de sujets très intimes. Dans le contact entre le médecin et le patient, l'importance de la confiance s'explique comme suit: au moment de l'anamnèse, le patient se trouve dans une position de faiblesse en raison d'un trouble physique et/ou psychique. De la confiance est nécessaire afin qu'un patient puisse s'ouvrir et répondre honnêtement à son médecin, même en cas de questions délicates. Tous les attributs dérivés, à savoir les connaissances spécialisées, l'amabilité, l'expérience professionnelle, l'empathie, la proactivité et la patience, jouent un rôle pertinent dans le développement de la confiance. Tous les attributs cités possèdent une connexion directe ou indirecte avec la confiance. Ce n'est que lorsque les patients ont confiance en leur médecin et s'ouvrent à lui que se développe un sentiment de bien-être et de sécurité.

\section{Références}

1 Neuenschwander P, Riedel M. Qu'est-ce que les patients aimeraient savoir sur leur médecin de famille? - Résultat d'une étude qualitative. Bulletin des médecins suisses. 2009;90:698-700.

2 Schläpfer L. Des médecins de famille qui répondent aux souhaits des patients. Bulletin des médecins suisses. 2010;91:1022-3.

3 Gruber T, Frugone F. Uncovering the desired qualities and behaviours of general practitioners (GPs) during medical (service recovery) encounters. Journal of Service Management. 2011;22:491-521.

4 Dieterich A. Arzt-Patient-Beziehung im Wandel: Eigenverantwortlich, informiert, anspruchsvoll. Deutsches Ärzteblatt. 2007;104:2489.

5 Letter K. Praxisführung: Den Patienten als Kritiker akzeptieren. Deutsches Ärzteblatt. 2010;107:1039-40.

6 Peters T. Macht im Kommunikationsgefälle: der Arzt und sein Patient. Band 82. Berlin: Frank \& Timme GmbH Verlag; 2008.

7 Diviani-Preiswerk S. Kommunikation in der Praxis: Wie sag ich's meinen Patienten? Bulletin des médecins suisses. 2014;95:616-8.

8 Donner-Banzhoff N. Arzt und Patient: Archäologie einer Beziehung. Deutsches Ärzteblatt. 2012;109:2078.

Correspondance:

Dr Carolin Dengler-Voss

Fachärztin FMH für Allgemeine Innere Medizin

Verkehrsmedizinerin SGRM

Universität Zürich, Institut für Rechtsmedizin -

Abteilung Verkehrsmedizin \& forensische Psychiatrie

carolin.dengler-voss[at]irm.uzh.ch 